\title{
EFFECTIVE MASS DETERMINATION OF A HIGHLY DOPED InAS-GaSb SUPERLATTICE USING HELICON WAVE PROPAGATION
}

\author{
J.C. MAAN \\ Max-Planck-Institut für Festkörperforschung, Hochfeld-Magnetiabor, $166 x, 1,38042$ Grenohle Cedex, \\ France
}

\author{
M. ALTARELLI \\ Max-Planck-Institut für Festkörperforschung. Stutgart. Fed, Rep, of Germany
}

\section{H. SIGG and P. WYDER}

Resedrch Institute for Materials, University of Nijmegen. Toernooiveld, Nijmegen, The Netherlonds and

\author{
L.L. CHANG and L. ESAKI \\ IBM. Thomas J. Watson Research Center, Yorktown Heights, New York 10598, USA
}

Received 24 August 1981; accepted for publication 4 September 1981

The far infrared radiation transmission of a highly doped InAs-CiaSb superlattice as a funclion of the magnetic field, shows helicon wave propagation. The effective mass and the carrier density are determined from an analysis of the results as a function of frequency to be $0.082 \pm 0.005 m_{0}$ and $3.4 \times 10^{18} \mathrm{~cm}^{-3}$. The carrier density is equal to that obtained from Hall measurements. The effective mass is significantly higher than the value expected from the InAs conduction band nonparabolicity $\left(0.063 m_{0}\right)$.

Several experiments have confirmed simple calculations of the subband structure and the effective mass in InAs-GaSb superlattices for the lowest electron subbands [1-3]. There is, however, much less experimental information about the subbands at higher energy. Such information is interesting because of the specific character of the formation of subbands in InAs-GaSb superlattices $[4,5]$. It has been established that the band line-up between InAs and $\mathrm{GaSb}$ is such that the GaSb-VB (valence band) is about $150 \mathrm{meV}$ higher than the InAs-CB (conduction band). Experimental facts about the lowest subbands therefore mainly probe the energy region where the bulk material bands would overlap, whereas the higher energy subbands are located in the energy gap of $\mathrm{GaSb}$. 
To obtain information about the mass at higher energies in InAs-GaSh superlattices, magnetotransmission experiments were performed on highly n-lype doped samples (free carrier density $n=3.4 \times 10^{18} \mathrm{~cm}{ }^{3}$ ). Using the calculated subband energies and integrating over the density of states a Fermi energy of $340 \mathrm{meV}$ is obtained which is $90 \mathrm{meV}$ above the original bulk $\mathrm{GaSb}-\mathrm{VB}$ edge and therefore in the band gap of GaSb. The effective mass is determined from the analysis of the transmission as a function of frequency and magnetic field. which shows helicon wave propagation in the superlattice.

Table 1 summarizes the properties of the experimental sample. The subbands are calculated by matching the InAs-CB and GaSb-VB and their derivatives at the interface in the two band $k \cdot p$ model as described in ref. [4]. In the calculation $0.023 m_{0}$ and $0.052 m_{0}$ are used for the effective masses of the InAs-CB and GaSb-VB respectively. The band gaps are $0.41 \mathrm{eV}$ for InAs and $0.81 \mathrm{eV}$ for GaSb. The energy difference of the GaSb-VB edge to the InAs-CB edge is taken as $0.15 \mathrm{eV}$.

Experimentally the transmission at fixed photon energy is measured as a function of the magnetic field, or alternatively at fixed magnetic field as a function of frequency. For the measurements at fixed photon energy an optically pumped far infrared laser was used as a source and the field was generated with a $5 \mathrm{MW}, 15 \mathrm{~T}$ Bitter coil. The measurements at fixed magnetic field were done with a superconductive magnet and a Michelson interferometer. The sample temperature was $10 \mathrm{~K}$ for the measurement with the laser and $1.5 \mathrm{~K}$ for the measurements with the Michelson interferometer. No temperature dependence was observed in the data between 1.5 and $40 \mathrm{~K}$.

Fig. 1 shows typical results of the experiments at fixed frequency. For all frequencies an increase in transmission is observed at increasing magnetic field. The transmission reaches a maximum at a certain magnetic field and decreases at higher field. The transmission maximum is at the low field side accompanied by a shoulder, which is clearly visible at high photon energies. Both the position of the shoulder and the maximum depend on the photon energy. The increase in transmission is quite drastic; for a radiation energy of $12.0 \mathrm{meV}$ a factor 60 transmission increase is observed in the maximum

\section{Table 1}

Layer thickness InAs

Laver thickness GaSb

Number of layers

Carrier density (Hall effect)

Mobility

Electron subbands

Fermi energy (above lowest subband)

$$
\begin{aligned}
& 150 \mathrm{~A} \\
& 150 \mathrm{~A} \\
& 67 \\
& 3.4 \times 10^{14} \mathrm{~cm}^{3} \\
& 14000 \mathrm{~cm}^{2} / \mathrm{V}^{3} \\
& 58 \mathrm{meV} \\
& 178 \mathrm{meV} \\
& 308 \mathrm{meV} \\
& 286 \mathrm{meV}
\end{aligned}
$$




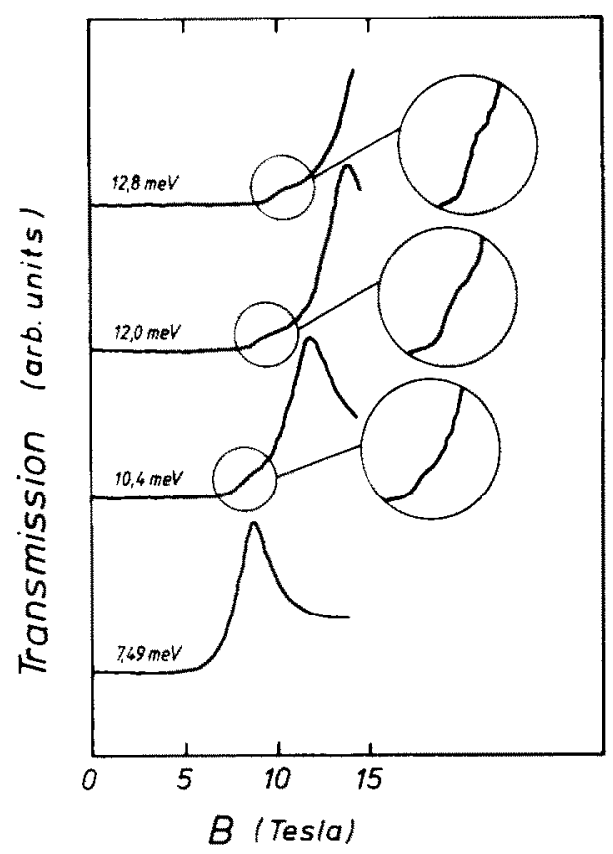

Fig. 1. Far infrared radiation transmission of the sample as a function of the magnetic field for different radiation energies. The magnetic field is perpendicular to the layers and parallel to the propagation direction of the radiation. The insets show an amplification of the shoulder at the low field side of the peak.

compared with the zero field transmission. Fig. 2 shows the magnetic field position of the maximum and the associated shoulder as a function of the radiation energy. The data from the interferometer are also included. The figure shows a linear relation between the position of the transmission maxima and the radiation energy, which extrapolates to zero field for zero energy.

These results can be explained as helicon wave propagation inside the superlattice sample, which has $2 \mu \mathrm{m}$ total thickness. The refractive index of the magnetoplasma inside the superlattice is a function of the magnetic field, therefore interference occurs when the wavelength in the material is an integral number $N$ times the superlattice thickness, giving rise to an oscillating transmission as a function of the magnetic field. Therefore a transmission maximum occurs for a magnetic field at which $\lambda / 2 \sqrt{ } \epsilon$, with $\lambda$ the far infrared wavelength and $\epsilon$ the dielectric constant of the medium, equals $d / N$ where $d$ is the superlattice total thickness and $N$ an integer. Using standard [6] formula for the response function of a plasma in a magnetic field under the conditions that the cyclotron frequency $\omega_{c}$ is of the order of the experimental frequency $\omega$, much smaller than the plasma frequency $\omega_{\mathrm{p}}$ and much higher than the collision frequency $1 / \tau(\tau$ the scattering time), the dielectric constant of the magneto- 


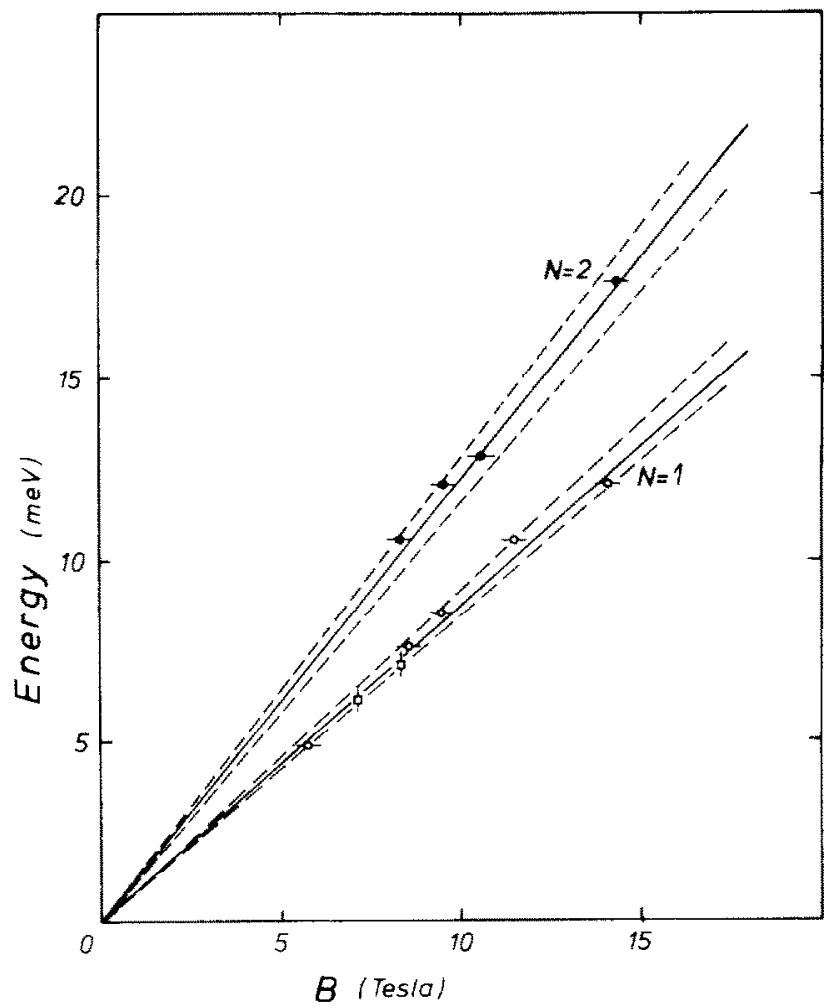

Fig. 2. Energy positions of the maximum and the shoulder in the transmiosion spectra as a function of the magnetic field: circles. far infrared laser data: squares, interferometer datit. The wlid lins: represent a theoretical fit with $m^{*}-0.082 m_{0}$ and $n=3.4 \therefore 10^{1 *}$ cm ${ }^{3}$, white the dotted lines illustrate the cror margin between the fits for different walues of $m^{*}$ and $n$ as discussed in the text. The two series correspond to photon wavelength in the medium cqual to $(1) 2$ or twite $(1)$ the total thickness of the superlatices.

plasma is given by:

$\epsilon=\epsilon_{\mathrm{r}}\left[1-\omega_{\mathrm{p}}^{2} / \omega\left(\omega \pm \omega_{\mathrm{r}}\right)\right]$.

Here $\epsilon_{\mathrm{r}}$ is the statistical dielectric constant of the medium, the + sign corresponds to the cyclotron resonance inactive (CRI) and the - sign to the cyclotron resonance active (CRA) polarization mode of the radiation. Propagation will occur as $\epsilon>0$ and this can only take place for the CRA mode as $\omega_{c}>\omega$. Considering further only the CRA mode the condition for a transmission maximum can be written as:

$$
\frac{B}{\omega}=\frac{m^{*}}{e}\left(1+\frac{n e^{2} d^{2}}{m^{*} \epsilon_{9} N^{2} \pi^{2} c^{2}}\right) \text {. }
$$


with $B$ the magnetic field, $m^{*}$ the effective mass, $n$ the free carrier density, $\epsilon_{0}$ the permeability of free space, $e$ the electron charge and $c$ the speed of light in vacuum.

The slope of the position of the transmission maximum as a function of $B$ and $\omega$ is determined experimentally and shown in fig. 2. From the two sets of maxima, which are assigned to helicon wave interference for $N-1$ and $N-2$ respectively, the effective mass and the carrier density can be obtained. The best fit of eq. (2) to the experimental data yields $n=3.4 \times 10^{18}$ and $m^{*}=$ $0.082 m_{0}$ ( $m_{0}$ is the free electron mass). The fit is shown as the straight line in fig. 2. The value for $n$ is the same as was obtained from Hall measurements (table 1). As can be seen from eq. (1), transmission occurs only for the CRA polarization mode. Therefore if the incident radiation is linear polarized the transmitted radiation will be circular polarized. This effect was experimentally verified.

It may come as a surprise that the volume carrier density is found because the superlattice consists of equally thick layers of $\mathrm{GaSb}$ which contain no free carriers, and InAs which contain twice the volume carrier density. As is clear from the previous analysis the wavelength in the medium at the transmission maximum is respectively twice and once the superlattice total thickness for $N=1$ and $N=2$. This wavelength is in both cases much longer than the individual layer thickness of $150 \AA$ and therefore only the overal carrier density is observed.

To evaluate the accuracy of the fit the two dotted lines in fig. 2 represent extreme cases with $n=2.8 \times 10^{18} \mathrm{~cm}^{-3}$ with $m^{*}=0.087 m_{0}$ and $n=4.4 \times 10^{18}$ $\mathrm{cm}^{-3}$ and $m^{*}=0.074 m_{0}$. Retaining the Hall value of $3.4 \times 10^{18} \mathrm{~cm}^{-3}$ for the carrier density, the effective mass can vary between $0.086 m_{0}$ and $0.077 m_{0}$.

It has been shown $[2,3,7]$ that the electron mass in InAs-GaSb superlattice is essentially given by the bulk InAs conduction band mass at an energy $E=E_{1}+E_{\mathrm{F}}$ with $E_{\mathrm{1}}$ the energy of the lowest conduction subband measured from the bulk InAs band edge and $E_{F}$ the Fermi energy. With $E=0.34 \mathrm{eV}$ for the present sample using the three band $k \cdot p$ model to calculate the energy dependence of the effective mass a value of $m^{*}=0.063 m_{0}$ is obtained, which is much lower than the value obtained from the experiments. It can easily be shown in a very direct way that a mass of $0.063 m_{0}$ is incompatible with the experimental data. As can be seen from eq. (1) the dielectric constant is negative for $\omega_{c}<\omega$. Therefore the onset of the transmission takes place at a magnetic field for which $\omega=\omega_{c}$, independent of the free electron density. For a mass of $0.063 m_{0}, \omega_{c}$ corresponds to a magnetic field where for a given frequency structure is already visible in the transmission. Consequently the mass must be higher.

In earlier work the effective mass of superlattices with a high Fermi energy due to either thin layers or heavy doping was determined through the temperature dependence of the Shubnikov-De Haas effect [7]. In this work also a mass enhancement which could not be explained by simple nonparabolicity was 
observed. The present experiments confirm this trent which at present is not understood.

A mass enhancement due to many body effects as was obesrved in Si MOS [8] devices is very improbable here as a consequence of the weakness of the electron-electron interaction; the kinetic energy of the electrons here is much higher both through the lighter effective mass and through the high Fermi energy. In ref. [7] it was suggested that the influence of dipole layers at the interface may play a role in the mass enhancement. However, the layer thicknesses here (150 $\AA$ ) are larger than that of the samples in ref. [7] (90 and 55 A) where a mass enhancement was observed, so these effects should be expected to be less important. To be able to make a more explicit conclusion about the origin of the mass enhancement more accurate bandstructure calculations of this type of superlattice than are available at present are needed.

In conclusion it has been shown that helicon wave propagation in InAsGaSb superlattices is possible and that this effect provides a useful method to measure the carrier density and the effective mass. The results from the experiments are in close agreement with $\mathrm{Hall}$ data for the carrier density. The observed mass of $0.082 \pm 0.005 m_{0}$ is much higher than expected from the InAs nonparabolicity.

\section{Acknowledgements}

Part of this work performed at the University of Nijmegen has been supported by the "Stichting voor Fundamenteel Onderzoek der Materie" (FOM) with financial support of the "Nederlandse Organisatie voor Zuiver Wetenschappelijk onderzoek" (ZWO). Work at the IBM Thomas J. Watson Research Center was partially supported under an Army Research Office Contract.

\section{References}

[1] For a recent review, see L.L. Chang and L.. Esaki. Surface Sci. 9x (1980) 70.

(2) H. Bluyssen. J.C. Maan, P. Wyder, L.L. Chang and L. Faaki, Solid State Commum. 31 (1979) 75 .

13) Y. Guldner. J.P. Vieren, P. Voisin, M. Voos, L.L. Chang and L. Lsaki, Phys. Rev. Letters 45 (1980) 1719.

(4) G.A. Sai-Halasz, R. Tsu and 1.. Ekaki, Appl. Phys Ledters 39 (1977) 651.

(5) G.A. Sai-Halasz, L. Esaki and W.A. Harrion, Phys. Rey. BI8 (1978) 2812.

16) R.S. Brazis, J.K. Furdyna and J.K. Pozela, Phys. Status Solidi (a) 53 (1979) II.

17) H. Sakaki, L.L. Chang, (i.A. Sai-Halasz, C.A. Chang and L. Esaki, Solid State Commun. 2f (1978) 589 .

[8] J.P. Kotthaus, Surface Sci. 73 (1978) 472. 